\title{
ESTIMATIVA DE SAFRA PARA A CULTURA DO MILHO ATRAVES DO FILME INFRAVERMELHO COLORIDO *
}

\author{
Gilberto J. Garcia ** \\ Delmar A. B. Marchetti ***
}

\section{RESUMO}

O sistema tradicional para estinativas de safra utiliza-se de relatórios técnicos de fontes diversas. A coleta de informações, se inicia antes da época do plantio e as estimativas são vendo ajustadas de acordo com o desenvolvimento das culturas. $O$ trabalho em questão, pretende mostrar as possibilidades do filme infravermelho colorido na estimativa de safra para a cultura do milho. As informações quantitativas foram obtidas através da densitometria de transmissão.

Os trabalhos foram conduzidos na Fazenda Experimental São Manuel da Faculdade de Ciências Agronômicas - Campus de Botucatu - UNESP.

Os seis tratamentos consistiram em doses diferenciais de adubo, prevendo prováveis quedas em produção. A cultura foi fotografada com filme colorido (Kodak Ektachrome 64 ASA) e infravermelho colorido (Kodak Ektachrome Infrared Film) e a densidade ótica medida em um microdensitômetro de transmissão marca Weston, modelo 877 e abertura de $0,8 \mathrm{~mm}$.

Para avaliar a interação entre Produção e Densidade Óíca, utilizou-se das análises de correlação e regressão linear. A análise dos resultados permitiram as seguintes conclusões principais: a) Desde que se faça as necessárias calibrações, o filme infravermelho pode ser utilizado na estimativa de safra para a cultura do milho; b) A utilização do densitômetro de transmissão, para a interpretação quantitativa dos resultados, revelou-se importante sugerindo a aplicação do método $\mathrm{em}$ ontros tipos de culturas.

\section{INTRODUÇÃO}

O sistema tradicional para estimativas de safra utiliza-se de relatórios técnicos, a partir de informações fornecidas pelos agricultores, pelas carteiras de crédito rural dos bancos, pelos órgãos de assistência técnica etc. A coleta de informações se inicia antes da época de plantio e as estimativas vão sendo ajustadas de acordo com o desenvolvimento das culturas. Embora mais sofisticada, a estimativa de safra

* Trabalho apresentado no VII Congresso Brasileiro de Engenharia Agrícola - Pelotas - RG - 1977. Entregue para publicação em 3-10-1977.

** Dept. ${ }^{\circ}$ de Engenharia Rural da Faculdade de Ciências Agronômicas de Botucatu - UNESP.

*** Dept. ${ }^{\circ}$ de Engenharia Rural da Escola Superior de Agricultura - "Luiz de Queiroz" USP. 
através do sensoriamento remoto, tem como principal vantagem a rapidez na obtenção das informações, além de reduzir substancialmente o volume de dados. Para as nossas condições, talvez uma fase intermediária seja a utilização de sensoriamento remoto para avaliar problemas culturais em micro regiões, em decorrência de geadas, secas, inundações, pragas, moléstias, etc.

Conforme comenta COLWELL (1970), o procedimento mais simples neste caso, seria medir a produção por unidade de área, de diferentes parcelas e relacionar estas produçôes com medidas fotométricas.

Existem no entanto diversas variáveis a afetar este relacionamento. GATES (1970), relata que a aparência das plantas e das superfícies vegetais, depende da sua interação com a radiação, sendo influenciada pela geometria das folhas, morfologia fisiologia, composição química, solo e clima. SPURR (1960) cita que as folhas coriáceas refletem menos que folhas suculentas e dentre os fatores internos a afetar a reflexão tem-se: variações na pigmentação, variações nas células do mesófilo, variação no teor de água e variação na epiderme das folhas.

GATE (1970) comenta ainda, que na região visível as folhas das diferentes espécies apresentam uma baixa refletividade não diferenciada, a qual cresce com o aumento do comprimento de onda, apresentando já na faixa pertencente ao infravermelho uma sensível distinção. Esta distinção na região infravermelha do espectro deve-se a quantidade de clorofila, evidenciando que a utilização de diferentes tipos de filmes na fotointerpretação da vegetação, acrescenta possibilidades adicionais ao analista.

Segundo FRITZ (1967), o filme Kodak Ektachrome Infrared Film é adequado dentre outras finalidades, para identificação de pragas e doenças em florestas, pomares e em culturas de cereais; identificação de espécies vegetais e no estudo das condições do solo. SCHULTE (1956) comenta que a técnica para se fotografar com filme infravermelho não difere muito da efetuada com filme comum. No entanto, duas providências são necessárias; a primeira refere-se ao uso de lentes apropriadas, visto que as lentes das câmaras convencionais são corrigidas de modo que os comprimentos de onda violeta e amarelo estejam ambos em foco, no mesmo plano. Se a câmara não for equipada com um dispositivo corretivo, próprio para filmes infravermelhos, uma correção aproximada deve ser feita, variando-se a distância focal em $0,25 \%$. A segunda providência é o uso de filtros, e conforme recomenda a Companhia Kodak (1972), o amarelo (wratten n. ${ }^{\circ}$ 12) deve ser necessariamente usado. Tal filtro bloqueia a luz azul, de tal modo que somente o verde, o vermelho e o infravermelho atingem a emulsão. Utilizando-se do filme infravermelho colorido, NORMAN e FRITZ (1965), MEYER e FRENCH (1967), MEYER e COLPOUZOS (1968) e 
PHILPOTTS e WALLEN (1969) obtiveram resultados consistentes na detecção de moléstias em diferentes espécies vegetais. GARCIA e MARCHETTI (1976) relataram a importância das informações obtidas através do filme infravermelho colorido no diagnóstico precoce de deficiência de nitrogênio em tomateiro. Três tratamentos, suficientemente repetidos, constituiram-se em doses diferenciais de nitrogênio. Aos 30, 50 e 70 dias após transplante foram obtidas fotografias coloridas de cada parcela ao mesmo tempo, concluindo-se que a fotografia infravermelha colorida registrou com antecipação de aproximadamente 15 dias as plantas que posteriormente apresentariam sintomas visuais de deficiência.

Embora evidenciada a importância do filme infravermelho, a interpretação qualitativa das fotografias sugere a necessidade de dados numéricos, passíveis deserem obtidos através dos densitômetros, que medem a densidade ótica. Segundo a COMPANHIA KODAK (1972), o densitômetro de transmissão é usado para medir a quantidade de luz que atravessa um filme, enquanto que o densitômetro de reflexão mede a quantidade de luz que reflete da superfície de uma cópia fotográfica.

A densitometria tem sido utilizada com sucesso por vários autores. MANZER e COOPER (1967) relataram que foi possível avaliar a severidade da sequência em uma cultura de batatas, através de medidas densitométricas em transferências infravermelhas coloridas. MURTHA (1969) comenta que foi possível detectar danos fisiológicos em coníferas através de medidas densitométricas, também em fotografias infravermelhas coloridas. JACKSON et alii (1971) obtiveram elevada corelação quando relacionaram medidas densitométricas de uma cultura de batatas com diferentes graus de infestação por requeima e níveis de infestação obtidos por patologias, para as mesmas parcelas no campo, evidenciando a utilidade do método.

O trabalho em questão, pretende mostrar as possibilidades do filme infravermelho colorido na estimativa de safra, para a cultura do milho. Neste caso, o filme colorido convencional constitui-se na testemunha. As informações quantitativas deverão ser obtidas por densitometria de transmissão.

\section{MATERIAL E MÉTODOS}

\subsection{Material}

Os trabalhos foram conduzidos na Fazenda Experimental São Manuel da Faculdade de Ciências Agronômicas - Campus de Botucatu — SP da Universidade Estadual Paulista "Júlio de Mesquita Filho".

A variedade de milho plantada foi a HMD 7974 peneira 20, em solo Latossolo Vermelho Escuro - fase arenosa. 
Os tratamentos em número de seis, consistiram em doses diferenciais de adubo, com o intuíto de evidenciar diferenças em produção, já encontradas em experimentos anteriores, com a cultura do milho. Cada tratamento contava com quatro repetições, cada uma com $60 \mathrm{~m}^{2}$.

Para obtenção das fotografias, utilizou-se de duas câmaras fotográficas marca Exakta Varex, com objetiva Tessar de $50 \mathrm{~mm}$. Uma das câmaras continha o filme colorido e a outra o filme infravermelho, mais o filtro Kodak Wratten n. ${ }^{\circ} 12$. Os filmes utilizados, são produzidos comercialmente pela Companhia Eastman Kodak. O filme colorido utilizado foi o Kodak Ektacrome 64 ASA, e o filme infravermelho colorido foi o Kodak Ektachrome Infrared Film, ambos em $35 \mathrm{~mm}$. A plataforma utilizada foi um avião monomotor tipo Paulistinha do Aero Club de Botucatu.

A densidade ótica foi medida por intermédio de um microdensitômetro marca Weston, modelo 877 , de transmissão, abertura de $0.8 \mathrm{~mm}$ e fonte de luz de $50 \mathrm{~W}$.

\subsection{Método}

Aos 90 dias após semeadura realizou-se um vôo no local, quando então se fotografou o experimento com os dois tipos de filmes. A altura de vôo mais adequada já era conhecida e foi de aproximadamente 130 metros (400 pés). As fotografias foram obtidas entre $10.00 \mathrm{e}$ 10.30 horas, sempre de um mesmo ângulo de tomada.

Revelados os filmes, estes foram analisados no microdensitômetro devidamente calibrado. Sendo pontual, foi possível realizar várias leituras densitométricas de cada repetição de $60 \mathrm{~m}^{2}$, de tal sorte que a leitura final constituiu-se na média de várias leituras parciais.

Para avaliar a interação entre Produção e Densidade ótica, utilizou-se das análises de correlação e regressão linear para os dois tipos de filmes. A significância do coeficiente de correlação e do coeficiente angular foi obtida através dos Testes " $F$ " $e$ " $t$ ", respectivamente. Para a análise estatística observou-se as recomendações contidas em PIMENTEL GOMES (1970).

\section{RESULTADOS E DISCUSSÃO}

Os valores da produção por tratamento e por parcela, para cada tipo de filme, bem como as respectivas leituras de densidade ótica são apresentados no QUADRO 1. Os resultados relativos a análise de correlação e regressão encontram-se no QUADRO 2, enquanto que a FIGURA 1 apresenta as respectivas retas ajustadas. 
QUADRO 1 - Produção de milho e densidade ótica dos filmes coloridos e infravermelho colorido.

\begin{tabular}{|c|c|c|c|c|}
\hline \multirow{2}{*}{ Trat. } & \multirow{2}{*}{ Parcelas } & \multirow{2}{*}{ Produç̃̃o (kg) } & \multicolumn{2}{|c|}{ Dens. Ótica } \\
\hline & & & I. V. & Col. \\
\hline \multirow{4}{*}{0} & 1 & 8,00 & 1,02 & 1,00 \\
\hline & 2 & 7,50 & 1,00 & 0,96 \\
\hline & 3 & 8,80 & 1,02 & 1,02 \\
\hline & 4 & 9,60 & 1,05 & 1,02 \\
\hline \multirow{4}{*}{1} & 1 & 10,00 & 1,19 & 1,04 \\
\hline & 2 & 11,50 & 1,22 & 1,08 \\
\hline & 3 & 9,80 & 1,18 & 0,98 \\
\hline & 4 & 9,30 & 1,15 & 1,04 \\
\hline \multirow{4}{*}{2} & 1 & 9,50 & 1,14 & 1,00 \\
\hline & 2 & 9,80 & 1,16 & 1,04 \\
\hline & 3 & 10,00 & 1,16 & 1,02 \\
\hline & 4 & 8,80 & 1,12 & 1,00 \\
\hline \multirow{4}{*}{3} & 1 & 9,20 & 1,10 & 1,01 \\
\hline & 2 & 9,00 & 1,10 & 1,01 \\
\hline & 3 & 8,70 & 0,90 & 1,00 \\
\hline & 4 & 9,70 & 1,20 & 1,03 \\
\hline \multirow{4}{*}{4} & 1 & 10,50 & 1,26 & 1,10 \\
\hline & 2 & 10,40 & 1,24 & 1,09 \\
\hline & 3 & 9,80 & 1,23 & 1,09 \\
\hline & 4 & 10,30 & 1,24 & 1,08 \\
\hline \multirow{4}{*}{5} & 1 & 6,50 & 0,95 & 1,00 \\
\hline & 2 & 7,00 & 0,98 & 0,98 \\
\hline & 3 & 6,00 & 0,94 & 0,97 \\
\hline & 4 & 6,90 & 0,97 & 0,98 \\
\hline
\end{tabular}

QUADRO 2 - Análise de correlação entre produção de milho por parcela e respectivas densidades óticas.

\begin{tabular}{lcccccc}
\hline $\begin{array}{l}\text { Tipo de } \\
\text { Filme }\end{array}$ & $\mathrm{A}$ & $\mathrm{B}$ & $\mathrm{R}$ & $\mathrm{R} \mathrm{R}^{2}$ & $\mathrm{TP}$ & $\mathrm{F}$ \\
\hline $\begin{array}{l}\text { I. Colorido } \\
\text { Colorido }\end{array}$ & 0,4894 & 0,0682 & $0,8641^{* *}$ & 0,7467 & $8,0536^{* *}$ & 64,8612 \\
\hline
\end{tabular}

* Significância ao nível de $1 \%$ de probabilidade. 

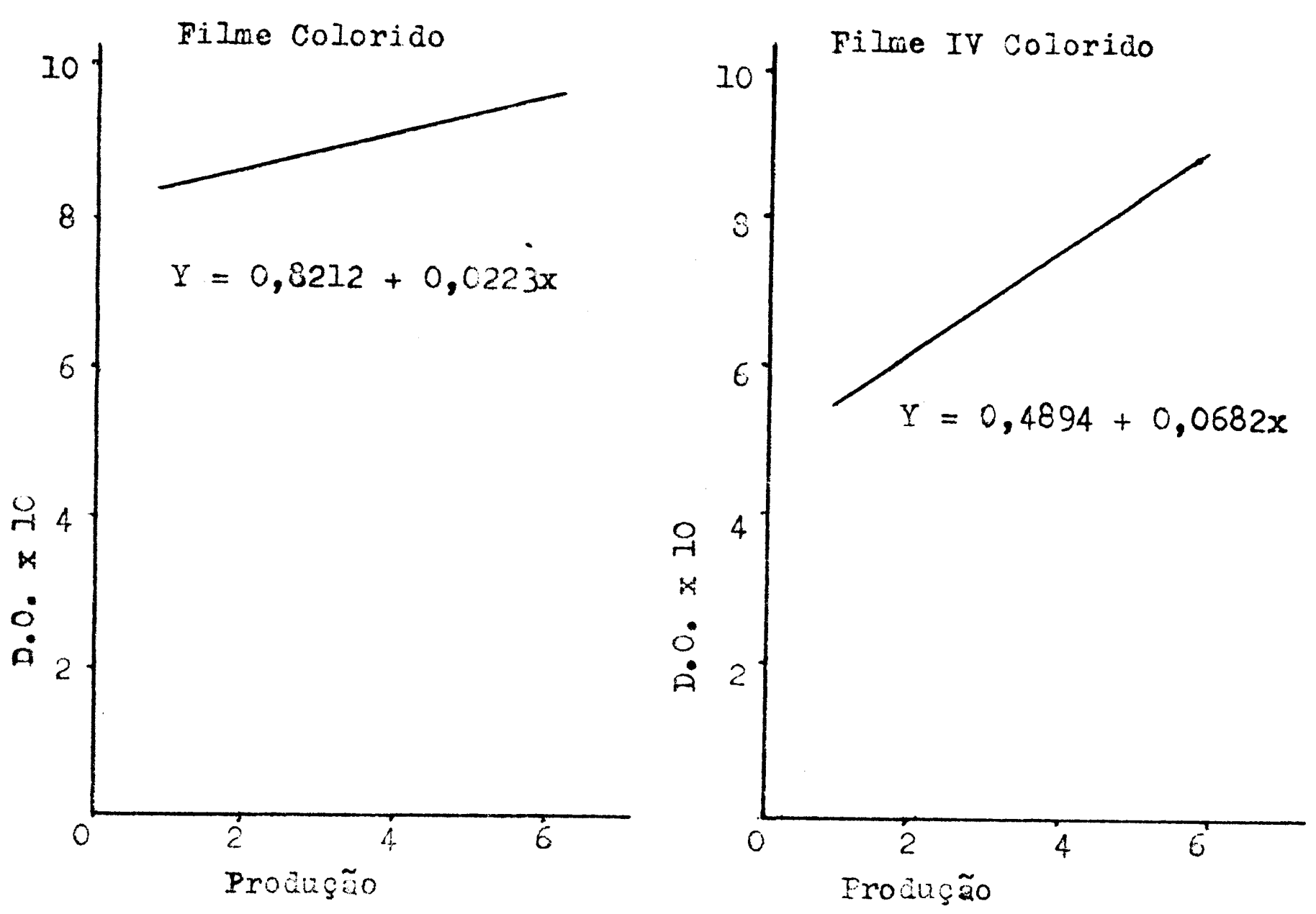

Figura 1 - Regressões entre produção e densidades óticas dos filmes colorido e
infravermelho colorido.

Para o filme infravermelho colorido o coeficiente de correlação foi de 0,86 com significância ao nível de $1 \%$ de probabilidade. Pela regressão obteve-se a equação $\mathrm{y}=0,4894+0,0682 \mathrm{x}$, com coeficiente angular significativo ao nível de $1 \%$ de probabilidade.

Para o filme colorido convencional o coeficiente de correlação foi de $0,77 \mathrm{com}$ significância ao nível de $1 \%$ de probabilidade. Pela regressão obteve-se a equação $\mathrm{y}=0,8212+0,0223 \mathrm{x}$, com coeficiente angular significativo ao nível de $1 \%$ de potencialidade.

Quanto ao coeficiente de correlação, embora em ambos os casos seja significativo, pode-se perceber que é mais elevado para o filme infravermelho colorido.

Quanto as análises de regressão, embora os coeficientes angulares sejam significativos, pode-se perceber pela FIGURA 1, que há uma maior interdependência de dados para o filme infravermelho colorido. Análises feitas com menor número de dados e menor amplitude de variação foram significativas para o filme infraveremhlo colorido e não significativas para o colorido comum, evidenciando a superioridade do primeiro. 
Análises feitas com menor número de dados e menor amplitude de variação foram significativas para o filme infravermelho colorido e não significativas para o colorido comum, evidenciando a superioridade do primeiro.

Os resultados obtidos são concordantes com GATES (1970) no que se refere a maior sensibilidade das medidas realizadas na região infravermelha do espectro.

Especificamente, quanto ao uso da densitometria de transmissão, o método revelou-se válido, concordando com MANZER e COOPER (1967), MURTHA (1969) e JACKSON et alii (1971), embora estes tenham estudado doenças em plantas. Em ambas as situações no entanto, são os desequilíbrios nutricionais presentes, que permitem diferenciações do registro espectral, em diferentes tipos de filmes. Assim, devidamente calibrados, os densitômetros podem fornecer valores de alta repetibilidade, evitando-se resultados provenientes de critério pessoal.

\section{CONCLUSÕES}

Para as condições estudadas pode-se concluir que:

a) desde que se faça as necessárias calibrações, o filme infravermelho pode ser utilizado na estimativa de safra para a cultura do milho.

b) a utilização do densitômetro de transmissão, para a interpretação quantitativa dos resultados, revelou-se importante, sugerindo a aplicação do método em outros tipos de culturas.

\section{SUMMARY}

\section{"CORN YIELD EVALUATION BY COLOR-INFRARED FILM"}

The traditional system of yeld forecasting utilizes technical reports from different resources. The collect of informations begins before the planting. period and the estimatives are adjusted during the development of the cultures. The present work, intends to show the possibilities of the color infrared film in the yeld forecasting of the corn. The quantitatives informations was obtained though the transmission density.

The work was conducted en São Manuel Experimental Farm of -Faculdade -de Ciências Agronômicas - Campus de Botucatu - UNESP.

The six trials consisted in different manure quantities, with probable yeld differences. The culture was photographed with color film (Kodak Ektachrome 64 ASA) and color infrared film Kodak Ektachrome Infrared Film) and the optical density was measured with a transmission microdensitometer, Weston, model 877 with a aperture of $0,8 \mathrm{~mm}$.

The interation between Yeld and Optica! Density was analysed through correlation and linear regression. The analysis of the results led to the follcwing main conclusions:

a) after the necessary calibrations, the color infrared film, can be used in the yeld forecasting of the corn;

b) the quantitative interpretation of the results through transmission density, suggest the application of this method in other types of cultures. 


\section{LITERATURA CITADA.}

COLWELL, R.N. Photogrammetric interpretation for civil purposes. In: American Society of Photogrammetry. Manual of Photogrammetry. Virginia. 1952. p. $535-602$.

COMPANHIA KODAK. Aplied infrared photography. N. York, 1972. 88 p. (Kodak Technical Publication M-28).

COMPANHIA KODAK. Pratical densitometry. N. York, 1972.15 p. ( odak Technical Publication E-59).

FRITZ, N.L. Optimum Methods for using infrared sensitive color films. Photogram. Engng., 33: 1128-1138. 1967.

GARCIA, G.J. e MARCHETTI, D.A.B. O filme infravermelho colorido (Falsa Cor) no diagnóstico precoce de deficiência de nitrogênio em tomateiro. Botucatu Científica. Série A, 1:27-30. 1976.

GATES, D.M. Physical and physiological properties of plants. In: National Academy of Sciences. Remote Sensing. Washington, 1970 p. 224-252.

JACKSON. H.R. et alii. Potato late blight intensity levels as determined by microdensitometer studies of false-color aerial photographs. J. Biol. Photogr. Assoc., 39: 101-106. 1971 .

8. MANZER, F.E. and COOPER, G.R. Aerial photographic methods of potato disease detection. University of Maine, Bulletin 646. 1967.

MEYER, M.P. and COLPOUZOS, L. Detection of crop diseases. Photogram. Engng., 34: 544-556. 1968.

MEYR, M.P. and FRENCH, D.N. Detection of disease trees. Photogram. Engng., 33: $1035-1040.1967$.

MURTHA, P.A. Near infrared detection of simulated animal damage on conifers. Proceedings of the workshop on aerial color photography in the plant sciences. Gainesville, Fla. 1969.

NORMAN, G. and FRITZ, N.L. Infrared photography as an indicator of disease and declive in citrus trees. Proc. Fla. State Hort. Soc., 78: 59-63. 1965.

PHILPOTTS, L.E. and WALLEN, V.R. IR color for crop disease identification. Photogram. Eng. 35: 1116-1125. 1969.

PIMENTEL GOMES, F. Curso de Estatística Experimental. São Paulo Nobel. 1970. $430 \mathrm{p}$.

SCHULTE, O.W. The use of panchromatic, infrared and color aerial photography in the study of plant distribution. Photogram. Engng. 17:688-714. 1951.

SPURR, S.H. Photogrammetry and Photo Interpretation. N. York, Ronald Press. 1960. 472 p. 